A reflection on cognitive reflection - testing convergent validity of two versions of the Cognitive Reflection Test

\author{
Nikola Erceg ${ }^{1}, Z_{\text {vonimir Galić}{ }^{1}, \text { Mitja Ružojčić }}{ }^{1}$ \\ ${ }^{1}$ Faculty of humanities and social sciences, University of Zagreb, Croatia
}

This work is a part of the project "Implicit personality, decision making and organizational leadership" funded by the Croatian science foundation.

Data can be found here: https://osf.io/zctme/

This article is a preprint and it has not yet been published in a peer-reviewed journal. Comments, feedback, ideas, critiques, suggestions are very welcome. Feel free to address them to Nikola Erceg.

Email: nerceg@ffzg.hr, Twitter handle: @nertzeg 


\begin{abstract}
Although it is generally acknowledged that the Cognitive Reflection Test (CRT) captures intelligence and numerical ability, many agree that it cannot be completely reduced to these constructs. Rather, it is presumed that the CRT also assesses some kind of thinking disposition towards reflective and open-minded thinking. In this manuscript, we report the results of a study that tested this assumption by exploring convergent validity of both the numerical and verbal version of the CRT. Using structural equation modelling, we investigated whether intelligence and numerical ability can account for all the variance in the CRT and if not, what is the nature of the unaccounted variance. Our conclusions about the convergent validity differed for the two types of test. For the numerical CRT, we found that the correlation between the latent numerical CRT and numerical ability was so high that the constructs were practically indistinguishable. As for the verbal CRT, the correlations between the latent verbal CRT and intelligence and numerical ability constructs were substantially lower, meaning that these two constructs do not account for all the variance in the test. However, the latent verbal CRT failed to correlate with belief bias and actively open-minded thinking, two closely related constructs, once the variance of intelligence and numerical ability was partialled out. We concluded that, despite its name, the CRT does not seem to assess the construct of cognitive reflection and its correlation with other variables found in the literature might mostly be driven by its overlap with intelligence and numerical ability.
\end{abstract}

Keywords: Cognitive reflection; Convergent validity; Numerical ability; 


\section{A reflection on cognitive reflection - testing convergent validity of two versions of the Cognitive Reflection Test}

\section{Introduction}

Reflection is defined as a „serious and careful thought" by the Cambridge dictionary and a „consideration of some subject matter, idea, or purpose“ by the Merriem - Webster dictionary. This definition of reflection implies reflection is different from intuitive or impulsive thinking and judging. Frederick (2005) constructed a short three-item measure in which every question was designed in a way that triggers an intuitive, impulsive answer that is always incorrect. In order to resist reporting the (inaccurate) response that first comes to mind, it is presumed that a person needs to ,reflect" on it and engage in slower and more deliberate thinking that is required to realize the correct response. Because of this characteristic, the test was named a Cognitive reflection test (CRT). In his seminal paper, Frederick reported that for the majority of students the CRT was quite hard, in spite the fact that it requires only basic mathematical skills to correctly solve the problems. CRT was also related to different measures of cognitive abilities and analytic cognitive style, but the correlations were low enough to allow the conclusion that the CRT and other used cognitive measures „likely reflect common factors, but may also measure distinct characteristics, as they purport to“"(Frederick, 2005, p. 35).

Since then, the CRT became popular among researchers because of its brevity but also due to the fact that it was able to predict an incredibly wide range of cognitive and behavioral outcomes. Specifically, CRT has been found to predict a performance on a range of tasks from the heuristics and biases domain. For example, the CRT score was negatively correlated with susceptibility to the conjunction fallacy and conservatism in updating probabilities (Oechssler, Roider \& Schmitz, 2009), the base rate fallacy (Hoppe \& Kusterer, 2011), the belief bias as measured by the belief bias syllogisms (Bubić \& Erceg, 2015) and positively correlated with a general indicator of resilience on using mental shortcuts as indicated with a composite of 15 different heuristics and biases tasks, including sample size problem, gambler's fallacy, bayesian reasoning, framing problem, sunk cost and others (Toplak, West \& Stanovich, 2011). Moreover, the predictiveness of the CRT spans outside the cognitive domain. CRT was found to be a significant predictor of religious belief (Pennycook, Cheyne, Seli, Koehler, and Fugelsang 2012; Shenhav, Rand \& 
Greene, 2012), political orientation (Deppe et al., 2015; Pennycook \& Rand, 2019), science understanding (Shtulman \& McCallum, 2014, Gervais, 2015), moral reasoning (Paxton, Ungar \& Greene, 2012; Royzman, Landy \& Goodwin, 2014) and susceptibility to pseudo-profound bullshit statements (Pennycook, Cheyne, Barr, Koehler \& Fugelsang, 2015; see Pennycook, Fugelsang and Koehler (2015) and Pennycook and Ross (2016) for a detailed account of predicitveness of the CRT across different domains).

Such breadth of the CRT bears the following question: where does this predictivity of the CRT come from? In other words, which construct(s) does give the CRT's its predictive power? Although researchers agree on some accounts, there are still substantial disagreements regarding the constructs the test captures. Despite the fact that most of the researchers agree that the CRT substantially taps into general cognitive ability, and numerical ability, there are still divergent opinions on whether or not it also taps into thinking dispositions that are, broadly defined, the tendencies towards particular patterns of intellectual behavior (e.g. the disposition to be intellectually careful, to seek and evaluate reasons, to be metacognitive etc.; Tishman \& Andrade, 1996). In order to come up with the correct answer on the CRT, a respondent first needs to reflect on the initial intuitive answer and recognize it is incorrect (reflection) but also to possess adequate cognitive ability and mathematical skills to calculate the correct response. Thus, it seems logical that the CRT should tap into both cognitive abilities and some disposition that predisposes a respondent to question the initial response. Thus, to put it simply, the question is whether or not the CRT taps into something that indicates an inclination towards reflective thinking once we control for the general cognitive ability and specific numerical ability.

\section{CRT and cognitive abilities}

There is a plethora of evidence that cognitive abilities play an important role in giving correct responses on the CRT. For example, in a recent study, Blacksmith, Yang, Behrend and Ruark (2019) found that the correlation between the latent CRT and latent $\mathrm{g}$ factor was very high $(\mathrm{r}=$ .74), near the cut-off value that indicates absence of discriminant validity (Shaffer, DeGeest \& Li, 2016). The authors concluded that the CRT likely captures a lower order intelligence factor, likely quantitative ability. In the initial article on the CRT, Frederick (2005) also reported reasonably high overlap with a measure of general intelligence (Wonderlic Personnel Test) and SAT scores 
that were used as a proxy for cognitive ability (the correlations of CRT with the two variables were .43 and 44, respectively). The correlations were probably underestimated because the CRT is short and relatively unreliable measure. Therefore, it is understandable that some researchers claim that CRT results mainly reflect individuals' general intelligence (e.g. Gino \& Ariely, 2012).

Research indicated that accurate responses on the CRT also correlated with numerical ability. Studies that simultaneously assessed CRT scores and numeracy found that the two scores were highly correlated. For example, Liberali, Reyna, Furlan, Stein \& Pardo (2012) reported the correlation of $r=.55$ and $r=.39$ between the numeracy and CRT across two studies. Similar correlations between the two constructs $(r=.40$ to .60$)$ were also reported in other studies (Campitelli \& Gerrans, 2014; Finucane \& Gullion, 2010; Primi et al., 2016; Thomson \& Oppenheimer, 2016; Welsh, Burns and Delfabbro, 2013). However, considering that the reported correlations were not dissatenuated and that both CRT and numeracy had moderate levels of internal consistency, they might have underestimated the exact size of the relation due to measurement error. The only disattenuated correlation we found in the literature is from Thomson and Oppenheimer (2016) who reported a very high correlation between the CRT and numeracy of $\mathrm{r}=.86$.

There are several additional streams of evidence that numeracy plays a key role in explaining predictiveness of the CRT. The first one comes from studies that utilize different scoring techniques of the CRT. ( e.g., Erceg \& Bubić, 2017; Pennycook, Cheyne, Koehler \& Fugelsang 2015). Specifically, CRT can be scored separately for indicators of reflection and numeracy. For example, as a reflection score, it is possible to calculate whether or not a respondent gave intuitive answer regardless of whether or not the answer was correct. This is sensible approach as a person can be reflective enough to recognize that the intuitive response is incorrect and, thus, resist reporting it. However, this still does not mean that the person is numerate enough as to be able to calculate the correct response. Therefore, as a measure of numeracy, it is possible to calculate, out of all the responses on which the intuitive response has not been given, how many responses were actually correct. Sinayev and Peters (2015) showed that only the CRT numeracy indicator predicted accurate responding on decision-making tasks while the CRT reflection indicator did not provide additional predictive power. This lead the authors to conclude that the ,results support the 
numeracy hypothesis, which posits that individuals with greater numerical ability will demonstrate fewer decision biases and achieve better financial outcomes, and it will account for the predictive power of cognitive reflection" (p. 12). Similarly, Campitelli and Gerrans (2014) showed that the CRT numeracy indicator and additional measure of numerical abilities were closely tied. Namely, for one standard deviation increase in the numerical ability score, the odds of calculating a CRT in a correct way was tripled or even quadrupled. In their study, the numerical abilities were the most important predictor of accurate responding on the CRT in a battery of rational thinking ability and thinking disposition measures.

The other stream of evidence for the importance of numeracy in successful responding on the CRT comes from the studies that used factor analysis to investigatethe relationships between the CRT and numerical ability measures. For example, Weller et al. (2013) factor-analyzed several numeracy scales together with the CRT items and, on the basis of high loadings on a single factor and high reliability, developed a new 8-item numeracy scale that included two out of the three original CRT items. Similarly, in Liberali et al. (2012) and Baron, Scott, Fincher and Metz (2015), CRT loaded on the same factor as did several other numeracy items. Thus, Baron et al. (2015) concluded that there is no evidence that "intuitive lures" matter at all for reliability or predictive validity of the CRT. A final piece of evidence that numerical ability is crucial prerequisite for the CRT success comes from the study by Stupple, Pitchford, Ball, Hunt and Steel (2017) who examined a time course of solving the CRT items. They showed that the response times were very weakly related with the accuracy on the CRT problems, leading them to the conclusion that people are not „cognitive misers” but ,cognitive wastrels”, meaning that they ,seem to be trying to compute a response whilst not possessing sufficient cognitive abilities - numeric or otherwise - to derive an effective solution" (p. 13).

\section{CRT and cognitive reflection}

Our short review reveals that there is quite a number of studies indicating that the CRT largely captures cognitive and/or numerical ability. Despite this, there are still disagreements on whether it also taps into thinking dispositions. Given the design of the CRT tasks, although respondents needs to possess adequate computational power and mathematical abilities to calculate the correct response, first they needs to reflect on the initial intuitive answer and recognize it is incorrect. 
Therefore, it seems logical that, alongside cognitive/numerical abilities, the CRT to a certain extent also taps into a disposition that predisposes respondents to question the initial response. To put it simply, the question is whether the CRT taps into something that resembles reflective thinking once we control for general cognitive ability and specific numerical ability.

Plenty of studies investigated whether the CRT assesses something beyond intelligence and numerical abilities. For example, Shenav et al. (2012) found that the CRT was related to belief in God even after controlling for several measures of cognitive abilities used in the study. Liberali et al. (2012) reported that the CRT was significant predictor of the score on the ratio bias task after controlling for numerical ability, while Toplak et al. (2011) showed that it predicted performance on a composite of tasks that assessed various cognitive biases after controlling for different intelligence measures. This "other construct" which differs from cognitive ability has been conceptualized as some kind of disposition related to cognitive reflection such as cognitive miserliness (Stupple et al., 2017; Toplak et al., 2011, 2014), cognitive impulsivity (Cokely \& Kelley, 2009; Graffeo, Polonio \& Bonini, 2015), actively open-minded thinking (Baron et al., 2015; Campitelli \& Labollita, 2010; Campitelli \& Gerrans, 2014) or analytic/intuitive cognitive style (Brosnan, Hollinworth, Antoniadou \& Lewton, 2014; Pennycook et al., 2012; Pennycook et al., 2015b; Pennycook \& Ross, 2016; Shenav et al., 2012).

Toplak et al. $(2011,2014)$ presume that the additional predictive power of CRT stems from the fact that CRT captures miserly processing, i.e. the disposition to default to heuristic processing mechanisms of low computational demands which is basically the opposite pole of reflection. Some evidence for this claim comes from the studies that reported a positive relationship between the response times and accuracy on the CRT items (e.g. Frey, Johnson \& De Neys, 2017; Stupple et al., 2017). The fact that the more time one takes to solve the CRT item the more accurate he/she is means that reflection could aid successful solving of CRT items. Others have conceptualized the additional variance unaccounted for by intelligence and numeracy as an actively open-minded thinking (AOT; Baron et al., 2015; Campitelli \& Labollita, 2010; Campitelli \& Gerrans, 2014). AOT can be defined as a set of dispositions that help a person avoid the tendency to confirm conclusions that are already strong and to search for more possibilities before making an inference (Baron et al., 2015; Campitelli \& Labollita, 2010). Thus, the willingness to question initial intuitive 
answers, one of the defining features of the CRT, is obviously an important element of the AOT. In sum, although most of the researchers agree that the CRT taps into cognitive abilities, especially numeracy, some believe that it also taps into some kind of thinking disposition.

\section{Our study}

Two important related questions arise from the previous discussion: a) Can the CRT be completely reduced to intelligence or specific numerical ability?; and b) If not, what is the nature of the variance in the CRT scores that remains unexplained by intelligence and numeracy measures? What does it capture? In the current study we tried to give an answer to these questions by using the regular numerical CRT and a newly developed verbal CRT (Sirota et al., 2018).To test the research questions we utilized a confirmatory factor analysis (CFA), the method that is considered to be the most appropriate for assessing discriminant validity of a construct (Shaffer et al., 2016).

Specifically, to answer the first question, we compared a model where the CRT and numeracy/intelligence loaded on a single latent factor with the model where each of them loaded on separate factors. If the models show similar fit to the data, it would suggest that the intelligence/numeracy accounted for all the variance in the CRT, i.e. that they essentially measure the same thing. However, if the one factor model shows a worse fit than the two factor model, this means that there is a substantial variance in the CRT still unaccounted for by intelligence/numeracy constructs. In this case, to examine the nature of this variance and respond to our second question, we examined models where CRT items loaded on the latent factors of AOT and belief-bias syllogisms (BBS), after controlling for the effects of intelligence/numeracy. These loadings will give us an insight into the nature of remaining variance in the CRT.

The AOT refers to a set of dispositions that oppose the tendency to confirm conclusions and beliefs that the person already holds and to search for more possibilities before making an inference (Baron et al., 2015; Campitelli \& Labollita, 2010). As we previously stated, it follows from this description that the very important element of the AOT is willingness to question initial intuitive answers. As this is also one of the defining features of the CRT, the AOT seems to be an appropriate measure for testing the convergent validity of the CRT after controlling for intelligence and numeracy. We also included BBS for two reasons. First, BBS and CRT are conceptually related as both of the 
tasks have a common characteristic that they cue an incorrect intuitive answer that needs to be detected and overturned by a correct, rule-based response. Therefore, BBS tasks should be appropriate for assessing miserly processing, i.e. the disposition towards defaulting to easier heuristic processing instead of engaging in a more deliberate reflection. Similarly as the CRT where one needs to overturn the urge to report the intuitive incorrect response in favor of the deliberate correct response, in BBS a solver needs to evaluate if the validity of a conclusion logically follows from premises instead of evaluating the conclusion based on its believability. A well-known syllogistic task goes as follows: "Premise 1: All flowers have petals. Premise 2: Roses have petals. Conclusion: Roses are flowers.” (Markovits \& Nantel, 1989). According to this syllogism, it does not follow that only flowers have petals, so roses might as well be something other than flowers (e.g. children collage art). However, because the conclusion that roses are flowers conforms with our empirical reality, it is quite believable and many people accept it as valid. Thus, the false intuitive response is the product of believability of the conclusion, while strong conformity with logical principles is needed to come up with the right, logically valid response. The tendency to evaluate conclusions based on believability instead of validity is called belief bias, and it is a welldocumented phenomenon (Evans, Barston, \& Pollard, 1983; Newman, Gibb, \& Thompson, 2017; Trippas et al., 2018).

Second reason for including BBS as a measure for assessing the convergent validity of the CRT is methodological. The BBS and CRT have been found to be correlated in number of research. The magnitudes of these correlations were quite high, for example $r=.36$ in Toplak et al. (2011), $r=$ .43 in Campitelli and Gerrans (2014) or $r=.56$ (disattenuated $r=.75$ in Thomson \& Oppenheimer, 2016). Given that the BBS tasks are not numerical, the magnitudes of these correlations point to the conclusion that the CRT and BBS might have something in common even after controlling for intelligence/numeracy. In fact, Baron et al. (2015) included both BBS and CRT items in their measure of overall cognitive reflection and concluded that they function well together and load highly on the same factor.

\section{Methods}

2.1. Participants 
506 university students participated in the study (27\% males). The mean age was 21.2 ( $\min =18$, $\max =31, \mathrm{SD}=2.13$ ).

\subsection{Instruments}

\section{a) Cognitive reflection}

We used two different CRT measures, the numerical one that required certain levels of mathematical skills to come to the correct responses and the verbal one that does not require any mathematical knowledge. We used an expanded, 10-item version of the classical CRT in order to increase reliability and response range of the total score. It consisted of three original CRT items (Frederick, 2005), but also additional items from previously reported alternative CRT measures (Primi et al., 2015; Thomson \& Oppenheimer, 2016; Toplak et al., 2014). An example of an item is "In an athletics team, tall members are three times more likely to win a medal than short members. This year, the team has won 60 medals so far. How many of these have been won by short athletes?". Here, the intuitive incorrect answer is 20 and the correct one is 15 . All the items

are listed in the Appendix. Total score was calculated by summing the correct responses, thus one could score anywhere between 0 (if none of the responses were correct) and 10 (if all the responses were correct).

CRT-verbal consisted of seven items taken from Sirota et al. (2018). An example of an item is "How many of each animal did Moses put on the ark?", where the intuitive response is two, but the correct one is zero (it was Noah that put animals on the ark). All the items are also listed in the Appendix. The total score was also calculated by summing up the correct responses.

\section{b) Intelligence}

We assessed intelligence with the International Cognitive Ability Resource (ICAR; for details see icar-project.com). ICAR is a broad cognitive ability assessment tool consisting of four different types of tasks: letters and numbers series, matrix reasoning items, verbal reasoning items and threedimensional rotation items. In this study we administered a 16 items version consisting of four items of each type. The validation of this measure is reported in Condon and Revelle (2014), and it was recently used by Blacksmith et al. (2019) in a similar context of CRT validation. 


\section{c) Numeracy}

We used The Berlin numeracy test (BNT; Cokely, Galesic, Schulz, Ghazal, \& Garcia-Retamero, 2012) as a measure of numeracy. The BNT is a four-question test for assessing numeracy and risk literacy. An example of a question is "Imagine we are throwing a five-sided die 50 times. On average, out of these 50 throws how many times would this five-sided die show an odd number (1, 3 or 5)?". The questions are designed in a way that they gradually become harder and a total score is calculated by summing up the correct responses on the four questions.

\section{d) Active open-minded thinking}

In this study we used a 15-item AOT scale introduced by Campitelli and Gerrans (2014). It is a self-report scale where participants indicate their level of agreement with the items on a six-point scale ( 1 - strongly disagree to 6 - strongly agree). An example of an item is "It is OK to ignore evidence against your established beliefs". The total score on this scale is calculated as a mean level of agreement with the items and can be anything between 1 and 6 .

e) Belief-bias syllogisms

BBS tasks assess the susceptibility to belief bias. In addition to the "Roses have petals" example we used three additional syllogisms whose conclusions were believable, albeit logically incorrect (see Appendix for all the tasks). We considered as correct the response where participants identified believable conclusion as logically incorrect. A participant's score ranged between 0 and 4 .

\subsection{Procedure}

Participants solved all the tasks as a part of a larger data collection effort in which they also solved a number of additional tasks that were not part of the current study. The regular and verbal CRT items were presented in four fixed, but different sequences and these sequences were randomly distributed across participants. All the other instruments were solved in fixed order. The students filled-in the tests and questionnaires on computers, in groups of 20 to 25 participants under the supervision of investigators. Participants were reimbursed with course credits and/or cinema gift cards. The whole testing session lasted up to two hours with a break of 10 to 15 minutes in the middle of a session. Unfortunately, because we became aware of the verbal CRT when the data 
collection was already underway, only half of the sample $(\mathrm{N}=253)$ solved the verbal CRT. Thus, all the analyses related to the verbal CRT were conducted on half of the sample.

\section{Results}

Before presenting the results of the confirmatory factor analysis, in Table 1 we present the basic descriptive statistics and correlations among all of the variables.

Table 1. Descriptive statistics and correlations among all the variables. The reliabilities are shown in the diagonal, bivariate correlations are below the diagonal, disattenuated correlations are above the diagonal

\begin{tabular}{|c|c|c|c|c|c|c|c|c|c|c|}
\hline & $\mathrm{M}$ & SD & Min & Max & CRT & CRT-V & NUM & ICAR & BBS & AOT \\
\hline CRT & 5.59 & 2.91 & 0 & 10 & .92 & .46 & .74 & .63 & .59 & .25 \\
\hline CRT-V & 3.98 & 1.79 & 0 & 7 & $.39 *$ & .80 & .30 & .27 & .22 & .08 \\
\hline NUM & 1.56 & 1.12 & 0 & 4 & $.58^{*}$ & $.21 *$ & .67 & .55 & .51 & .23 \\
\hline ICAR & 10.29 & 3.09 & 1 & 16 & $.56^{*}$ & $.22 *$ & $.42 *$ & .85 & .44 & .26 \\
\hline BBS & 2.10 & 1.62 & 0 & 4 & $.55^{*}$ & $.19^{*}$ & $.40^{*}$ & $.39^{*}$ & .93 & .32 \\
\hline AOT & 4.51 & 0.65 & 1.87 & 6 & $.22 *$ & .07 & $.17 *$ & $.22 *$ & $.28 *$ & .82 \\
\hline
\end{tabular}

Note. ${ }^{*} \mathrm{p}<.05, \mathrm{p}<.01 ; \mathrm{CRT}$ - numerical CRT; CRT-V - verbal CRT; NUM - numeracy; ICAR intelligence; BBS - belief bias syllogisms; AOT - actively open-minded thinking;

\subsection{Numerical CRT}

\subsubsection{Intelligence vs. CRT}

To test if the cognitive reflection is distinguishable from intelligence, we compared two nested models, an unconstrained one in which latent factors of cognitive reflection and intelligence were free to covary with a constrained one in which the correlation between the factors was fixed at 1 . If no difference between the models is found, then the more parsimonious model with greater degrees of freedom is retained, i.e. we can conclude that the cognitive reflection is not a different construct from intelligence. Before specifying the models, we first formed the subscale results by summing up the correct responses on the four items belonging to letters, numbers, matrix reasoning 
and verbal reasoning subscales. After that, we fitted models where the ICAR subscale scores were manifest variables for the ICAR latent factor and the CRT items were manifest variables for the cognitive reflection latent factor. The parameters and the fit of the model was estimated using diagonally weighted least squares (DWLS) as both ICAR and CRT variables were ordinal. Both of the models, one where the correlation between the latent factors was fixed at 1 and one where it was freely estimated, showed a good fit to the data $\left(\chi^{2}(77)=138.20, \mathrm{p}=.00 ; C F I=.98 ; T L I=\right.$ $.97 ; R M S E A=.04$ for the constrained model and $\chi^{2}(76)=119.97, p=.00 ; C F I=.98 ; T L I=.98$; RMSEA $=.03$ for the unconstrained model). However, when comparing the two models, the unconstrained model showed a significantly better fit than the constrained one $\left(\Delta \chi^{2}(1)=11.87, p\right.$ $=.00)$, although the estimated correlation between the factors was pretty high $(r=.82)$. The unconstrained model with factor loading and correlation between the factors is shown in Figure 1.

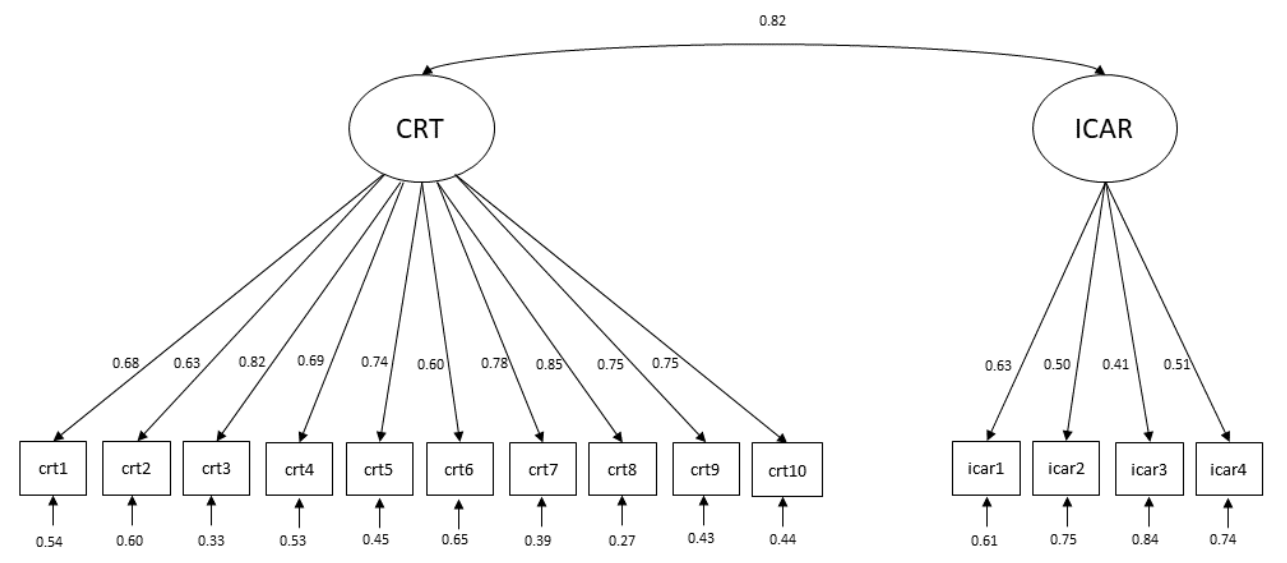

Figure 1. A two - factor model of cognitive reflection and ICAR

\subsubsection{Numeracy vs. CRT}

We repeated the same analysis to test the equivalence of the numeracy and cognitive reflection factors. We again fitted two models, a constrained one with the correlations between the factors fixed at 1 (one-factor model), and an unconstrained one with this parameter freely estimated (twofactors model). Again, both models showed very good fit to the data $\left(\chi^{2}(77)=104.48, p=.02 ; C F I\right.$ 
$=.99 ; T L I=.99 ; R M S E A=.03$ for the one-factor model and $\chi^{2}(76)=103.61, p=.02 ; C F I=.99$; $T L I=.99 ; R M S E A=.03$ for the two-factors model). However, this time there was no significant differences in the fit between the models $\left(\Delta \chi^{2}(1)=1.02, p=.31\right)$ indicating that the latent factor of cognitive reflection is practically indistinguishable from the latent factor of numeracy. This was further corroborated by the extremely high correlation of $r=.95$ that was estimated between the factors. This model is shown in Figure 2.

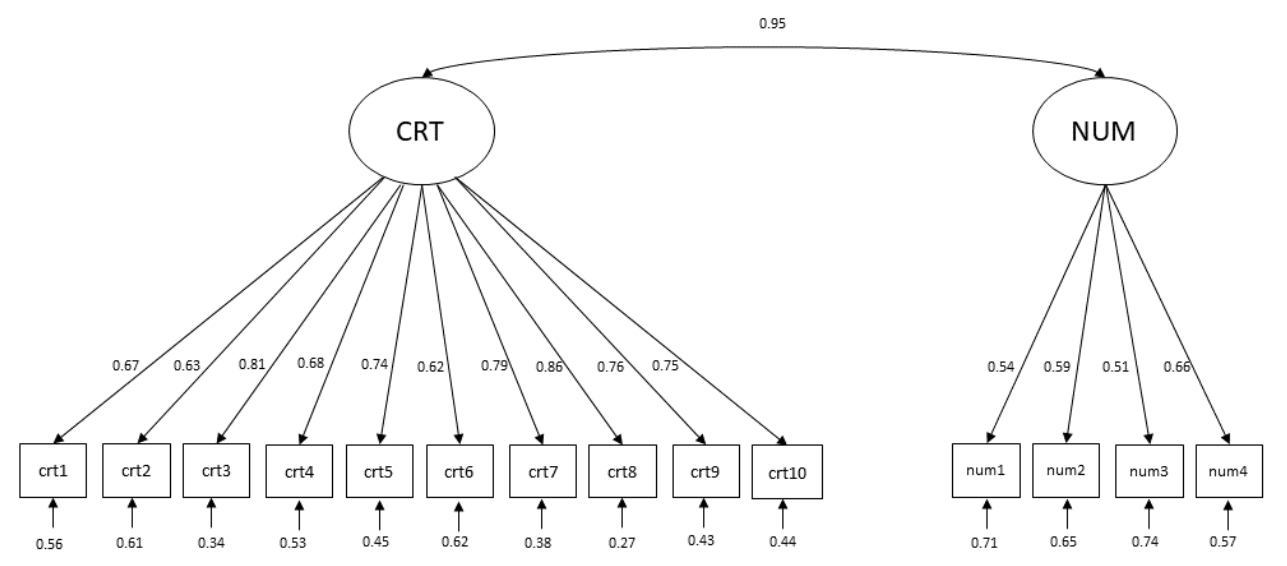

Figure 2. A two-factor model of cognitive reflection and numeracy

3.1.3. Does the latent CRT factor correlate with the BBS and AOT factors after partialling out the effects of general intelligence and numeracy?

To test for this possibility, we fitted two bifactor models. A bifactor model presumes that "the covariance among a set of item responses can be accounted for by a single general factor that reflects the common variance running among all scale items and group factors that reflect additional common variance among clusters of items, typically, with highly similar content." (Reise, 2012; p. 668). This general factor represents the conceptually broad construct that an instrument measures, and the group factors represent more conceptually narrow constructs. According to Reise (2012), the bifactor model is ideally suited for representing the multidimensionality that arises when broad constructs are measured with items from multiple and 
distinct domains. Thus, the bifactor model seems to be appropriate approach for testing the convergent validity of a specific group factor after partialling out the variance accounted for by a general factor.

Specifically, in both of the models that we fitted, we fitted a general factor ( $\mathrm{g}$ factor) that accounted for the shared variance in the CRT, the ICAR and the numeracy manifest variables. In addition, the CRT items also loaded on the second, group factor that accounted for the shared variance among the CRT manifest variables that was not accounted for by the $\mathrm{g}$ factor. Therefore, this factor should account for something that is not numeracy or general intelligence but is unique to the CRT items, perhaps cognitive reflection. Finally, we formed a latent BBS factor in the first model and the latent AOT factor in the second model, and allowed for the correlations between these factors and the latent CRT factor to be freely estimated (the correlation between the $\mathrm{g}$ factor and the CRT factor was set to be 0 as this is the way bifactor models are specified).

The first model where the latent CRT factor was correlated with the latent BBS factor showed an excellent fit to the data $\left(\chi^{2}(197)=200.13, p=.43 ; C F I=.999 ; T L I=.999 ; R M S E A=.01\right)$. The loadings of all the manifest variable on the $g$ factor were quite high (mostly above .5). On the contrary, the loadings of the CRT items on the CRT latent factor were generally low, except for the two items (crt2 and crt6) that had relatively high, but negative loadings. Most importantly, the correlation between the latent CRT factor and BBS factor was very low and non-significant $(r=-$ $.10, p=.27)$. In accordance to the earlier described CFAs, once the $\mathrm{g}$ factor is accounted for, CRT and BBS have very little in common and that their correlation is basically wholly driven by the correlation between the $\mathrm{g}$ factor and syllogisms (here estimated to be $r=.70, p=.00$ ). This model is shown in the Figure 3. 


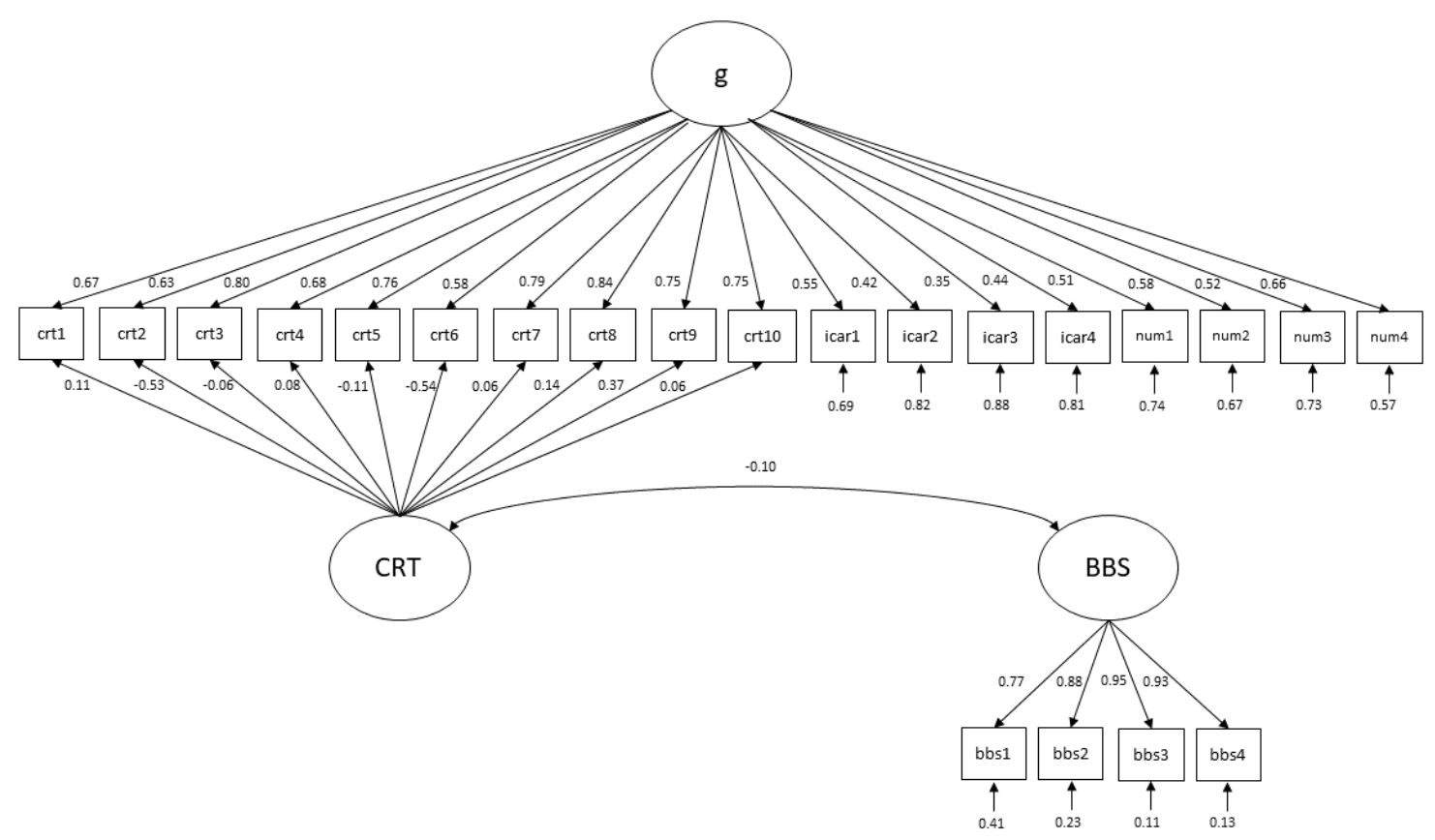

Figure 3. A bifactor model of the CRT with an estimated correlation between the CRT and the BBS factors

The second model where the latent CRT factor was correlated with the latent AOT factor showed very good fit to the data $\left(\chi^{2}(1483)=623.65, p=.000 ; C F I=.96 ; T L I=.96 ; R M S E A=.03\right)$. Again, the loadings of all the manifest variable on the g factor were quite high (mostly above .5). Similarly as with the first model, the loadings of the CRT items on the CRT latent factor were low, except for the crt6 item that again had relatively high, but negative loading. The small differences in loadings between the above model and this one stem from the fact that some participants had missing values on the AOT measure and the parameter values are, therefore, estimated on somewhat different samples $(\mathrm{N}=506$ for the model with the BBS and $\mathrm{N}=469$ for the model with the AOT). The correlation between the latent CRT and the latent AOT was somewhat higher that with the syllogisms, albeit still non-significant and negative $(r=-.20, p=.11)$. So the conclusion is again that the CRT has little in common with the AOT once the $\mathrm{g}$ factor is controlled for, and their generally positive correlation is mainly driven by the $\mathrm{g}$ factor $(r=.30, p=.00)$. This model is shown in the Figure 4. 


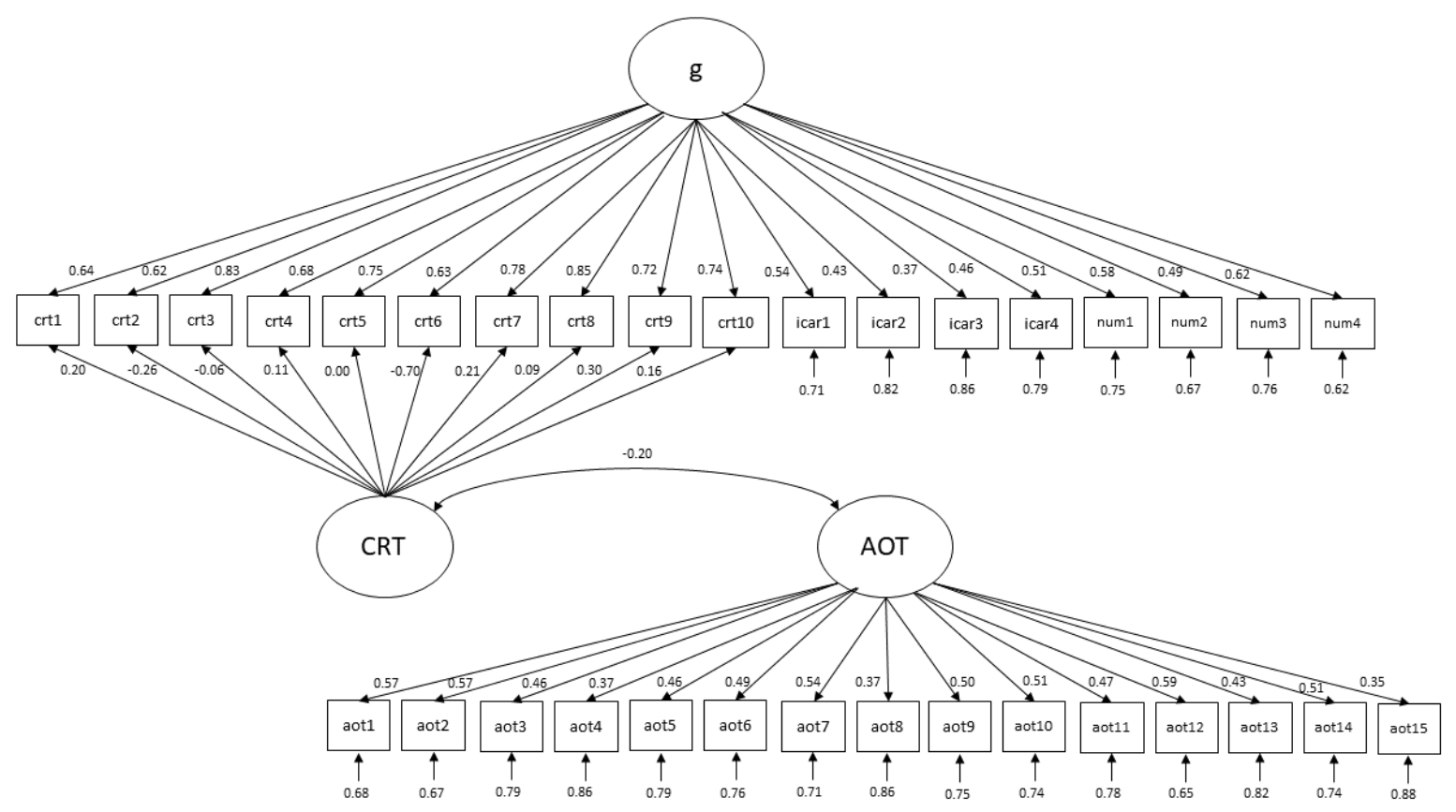

Figure 4. A bifactor model of the CRT with an estimated correlation between the CRT and the AOT factors

\subsection{Verbal CRT}

\subsubsection{CRT-V vs. intelligence}

To test whether or not the verbal cognitive reflection is distinguishable from intelligence we basically replicated the analysis we did with the regular CRT. Again, we compared the fits of the constrained and unconstrained models. The fit of the constrained model was below acceptable levels this time $\left(\chi^{2}(44)=89.55, p=.00 ; C F I=.85 ; T L I=.81 ; R M S E A=.07\right)$. However, the unconstrained model showed quite good fit to the data $\left(\chi^{2}(43)=49.63, p=.23 ; C F I=.98 ; T L I=\right.$ $.97 ; R M S E A=.03)$, significantly better than the constrained model $\left(\Delta \chi^{2}(1)=17.76, p=.00\right)$. The freely estimated correlations between the ICAR and the cognitive reflection factor was $r=.39$. This model is shown in Figure 5. 


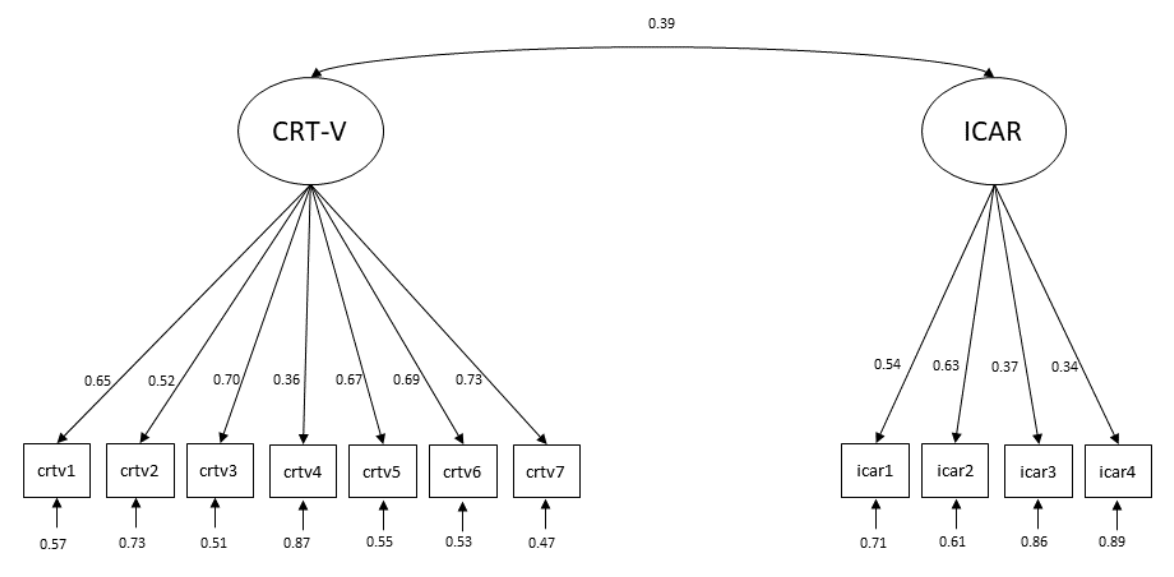

Figure 5. A two-factor model of verbal cognitive reflection and ICAR

\subsubsection{CRT-V vs. numeracy}

To test the relationship between the latent verbal cognitive reflection and the latent numeracy, we again fitted the two models where the relationship between the latent factors was either constrained at $r=1$ or unconstrained. The constrained model $\left(\chi^{2}(44)=72.52, p=.00 ; C F I=.90 ; T L I=.88\right.$; $R M S E A=.05)$ again showed a worse fit to the data than the unconstrained model $\left(\chi^{2}(43)=44.13\right.$, $\left.p=.42 ; C F I=.996 ; T L I=.995 ; R M S E A=.01 ; \Delta \chi^{2}(1)=15.91, p=.00\right)$ indicating that the verbal cognitive reflection and numeracy are not equivalent. The estimated correlation between the factors was $r=.39$. This model is shown in Figure 6. 


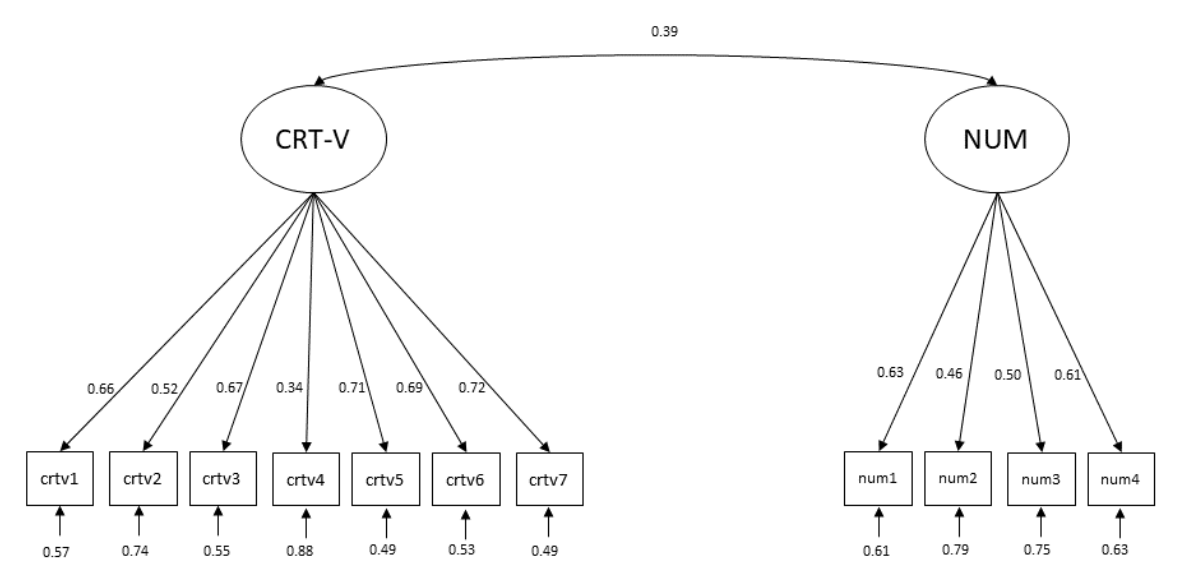

Figure 6. A two-factor model of cognitive reflection and numeracy

3.2.3. Does the latent CRT-V factor correlate with the BBS and AOT factors after partialling out the effects of general intelligence and numeracy?

Since it is obvious that the verbal cognitive reflection does not overlap with intelligence or numeracy, we wanted to examine the nature of this variance that was left unaccounted. In order to do that, we again fitted two models. Similarly as with the numerical CRT, in both models verbal CRT, ICAR and numeracy items loaded on the same general factor (g factor), with the verbal CRT items also loading on the CRT-V factor. Finally, the correlation between the CRT-V and the BBS factor (in the first model) and the CRT-V and the AOT factor (in the second model) was freely estimated.

The first model where the latent CRT-V and the latent BBS factors were correlated showed an excellent fit to the data $\left(\chi^{2}(143)=158.73, p=.17 ; C F I=.99 ; T L I=.99 ; R M S E A=.02\right)$. CRT-V items had somewhat lower loadings on the $\mathrm{g}$ factor then the numerical CRT items (mostly below .40), but much higher on the latent CRT-V factor (all except for the one above .50), which was expected as the latent CRT-V factor had substantial lower correlations with the ICAR and numeracy factors that the numerical CRT factor did. However, although there was substantial 
amount of variance in the CRT-V left unaccounted for by the $\mathrm{g}$ factor, this variance again had little to do with the BBS. Namely, the correlation between the latent CRT-V and the BBS factor was $r$ $=.06(p=.53)$, and the correlation between the verbal CRT and syllogisms was again fully driven by the correlation between the $\mathrm{g}$ factor and syllogisms $(r=.61, p=.00)$. This model is shown in the Figure 7.

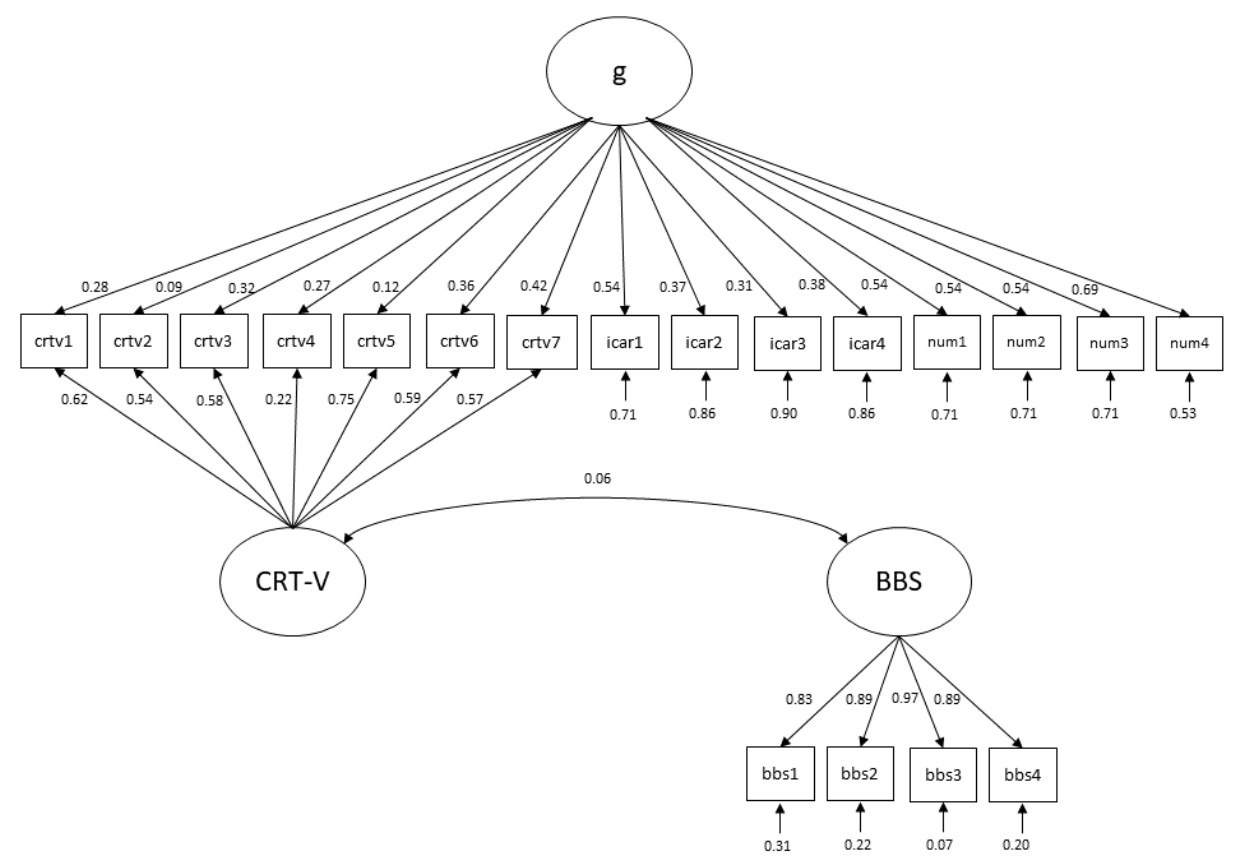

Figure 7. A bifactor model of the CRT-V with an estimated correlation between the CRT-V and the BBS factors

The second model where the latent CRT-V and AOT were correlated showed a good fit to the data $\left(\chi^{2}(396)=474.47, p=.004 ; C F I=.89 ; T L I=.88 ; R M S E A=.03\right)$. Again, the loading of the CRT$\mathrm{V}$ manifest variables on the g factor were relatively low (below .40), but on the latent CRT-V factor quite high (all except for the one above .50). However, the conclusion was again similar to the one above. The correlation between the latent CRT-V factor and the latent AOT factor was close to zero $(r=.04, p=.66)$, and the relationship between the CRT-V and the AOT is fully accounted for by the $\mathrm{g}$ factor (correlation between the $\mathrm{g}$ factor and the AOT was $r=.21, p=.00$ ). The model is shown in the Figure 8. 


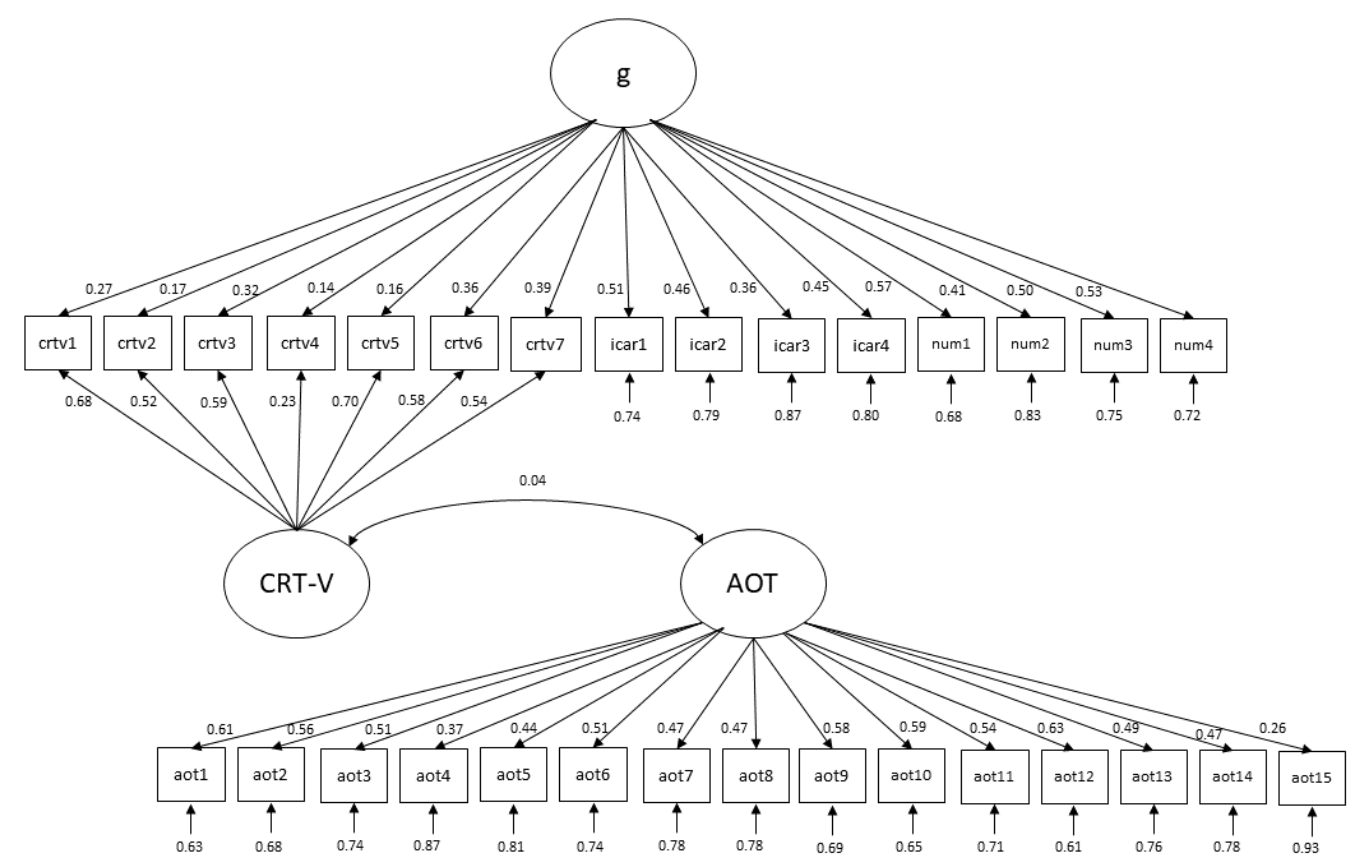

Figure 3. A bifactor model of the CRT with an estimated correlation between the CRT-V and the AOT factors

\section{Discussion}

The goal of this study was to explore convergent validity of the cognitive reflection construct. We did this by examining two versions of the CRT, a numerical CRT where mathematical knowledge is prerequisite for correct responding and the verbal CRT that does not require any mathematical knowledge. In particular, we wanted to see to what extent intelligence and numeracy account for reliable variance in these measures and, even more important, what is the nature of the CRT variance that was left unaccounted for (if there was any). Our findings were different for the two CRT tests. On the one hand, the results indicate that the cognitive reflection measured with regular math dependent CRT items was closely related to intelligence and practically indistinguishable from numeracy. On the other hand, cognitive reflection measured with verbal items seems to be a construct distinct both from the intelligence and numeracy. However, the nature of the variance in verbal CRT unaccounted for by the cognitive abilities is unclear. After partialling out the cognitive abilities, the CRT factor was not correlated with the belief bias and actively open-minded thinking factor, the two constructs that should be close to cognitive reflection in the construct's nomological network (Cronbach \& Meehl, 1955). This indicates that the unaccounted variance of the verbal 
CRT has little to do with cognitive miserliness or disposition to question own initial intuitive responses. In short, these results cast doubt on the ability of the cognitive reflection test to assess anything different from intelligence or numeracy or anything that would resemble cognitive reflection.

The most striking finding of the current study is very high correlation between the numerical cognitive reflection and numeracy factors that renders them practically indistinguishable. We see two possible explanations for this finding. First, the latent trait measured by the CRT is numerical ability and nothing else. This would support the position of researchers that conceptualize and treat the CRT as just another numeracy measure (Sinayev \& Peters, 2015; Weller et al., 2013; Welsh, Burns \& Delfabbro, 2013). It would also mean that predictiveness of the CRT for a range of outcomes described in the introduction stems mostly from numeracy. This would not be a novel idea. For example, Peters (2012) and Reyna, Nelson, Han and Dieckmann (2010) review a large body of literature showing how numeracy plays an important part in judgment and decision-making quality. In her article, Peters (2012) reviews studies in which greater numeracy has been associated with reduced susceptibility to framing effects, less influence of non-numerical information, greater sensitivity to numbers, and stronger, more precise number-related affect. Reyna et al. (2010) describe how the role of numeracy plays out in medical decision making, citing studies that show negative effects of low numeracy on perceptions of risks and benefits of screening, medication compliance, access to treatments, risk communication and resistance to extraneous factors that do not change objective numerical information.

However, the question is why are there studies that showed an incremental validity of the CRT beyond the pure numerical factor. The possible reason might lie in the numeracy instrument used in those studies. Namely, the most frequently used measure of numeracy in these studies is one developed by Lipkus, Samsa and Rimer (2001) that is arguably easier than both the CRT and Cokely et al. (2012) numeracy measure that we used in the current study. For example, the mean score on Lipkus et al. (2012) test was $\mathrm{M}=8.90$ and $\mathrm{M}=8.95$ out of 11 in Liberali et al. (2012) Study 1 and Study 2 respectively. The means in Primi et al. (2016) and Thomson and Oppenheimer (2016) were even higher ( $M=9.16$ and $M=9.23$ respectively). Therefore, it is possible that the CRT has additional variance beyond numeracy when numeracy is assessed with easier test but not 
with the more difficult one simply because it assesses a broader range of the latent numeracy trait, one that cannot be assessed with easy tasks. In other words, in order to successfully fight against the lures towards the wrong response in the CRT items, one does not need to be reflective but rather simply more numerate and mathematically proficient.

The alternative explanation for nearly perfect correlation between the cognitive reflection and numeracy factor is not that the CRT does not measure cognitive reflection but that both CRT and numeracy instrument asses it to some degree. If we return to the dictionary definitions of reflection provided at the beginning of the manuscript, it is not hard to see how both instruments could assess it. If reflection is defined as serious and careful thought then more serious and thoughtful approach to the two tests should grant more correct responses on both of them. However, in this case the CRT and similar tasks do not have exclusive status when it comes to measuring cognitive reflection and that construct should also be captured by other cognitive tasks that do not have a characteristic of cuing an intuitive wrong response. Also, this means that it would be very hard or impossible to uniquely capture a construct of cognitive reflection with any particular performance test and that other methodologies and measures should be used instead. The possibilities are combining time and accuracy measures (e.g. Baron et al., 2015) or using a two-response paradigm in which participants first give fast intuitive response, after which they reflect on it and can change it when giving their final response (e.g. Bago \& De Neys, 2017; Newman et al., 2017; Thomson, Turner \& Pennycook, 2011). However, it seems to us that in either case the cognitive reflection needs much more precise definition and conceptualization and more methodologically sound studies that would investigate the nature and validity of this construct. In pursuing these goals, researchers can use some of the excellent guidelines and resources available to them (e.g. Podsakoff, MacKenzie \& Podsakoff, 2016; Shaffer et al., 2016).

While the numerical CRT was practically in our study indistinguishable from numeracy, the correlations of the verbal CRT with intelligence and numeracy were significantly lower. Considering that one of the main reasons for developing the verbal CRT was to develop a cognitive reflection measure that will be much less dependent on the numeracy, in this regard the test met the expectations. However, even though majority of the variance in the verbal CRT is not accounted for by numeracy and intelligence, we still do not know what is the nature of this variance. The 
general problem with the verbal CRT in this study was that, despite adequate reliability, its correlations with conceptually related measures such as BBS and AOT were very low, substantially lower than those observed between the numerical CRT and these variables. Even those seems to be entirely driven by the $\mathrm{g}$ factor as the verbal CRT factor was not related to either BBS or AOT factor after partialling out the effects of $\mathrm{g}$ factor. Thus, although it seems that the verbal CRT measures something more than intelligence or numeracy, in the current study we did not figure out what that is. However, since it failed to show a relationship with variables closely conceptually related with CRT, we cannot be sure that it captures cognitive reflection. In sum, in our study we did not observe any convincing evidence that the two versions of CRT tests assess cognitive reflection defined as the disposition towards engaging in more deliberate reflection instead of defaulting to easier heuristic processing.

The conclusions of the current study are qualified by several facts. First, our sample consisted of college students that are on average more intelligent, numerate and open-minded than the general public. In this particular case, this fact can be relevant. Namely, college students have ample experience with basic mathematical operations that are required to successfully solve CRT items and through their education they had lots of opportunities to train their skills. This means that college students might have developed good mathematical intuitions that allow them to do basic mathematical operations swiftly and almost intuitively. It is also in line with the „hybrid” dualprocess model that posits that not only incorrect but also correct responses can be intuitively cued and with greater probability among those more experienced in particular task (De Neys, 2017). However, this could in turn mean that the effect of deliberation and reflection on accuracy in solving CRT tasks would be diminished in this sample. In other words, it could mean that the CRT is not particularly good measure of cognitive reflection for highly intelligent and numerate, thus additional studies with a more diverse sample in terms of education and cognitive abilities is welcome. The other significant drawback of the study is the fact that the sample for the validation of the verbal CRT was halved in comparison with full sample on which we validated a regular CRT. The reason for this was practical, due to time constraints. However, lower sample size could mean that the parameters are estimated with lesser precision. Finally, it must be noted that the verbal CRT measure is still in its nascent stage and that our study, as far as we are aware of, is the 
first attempt of cross-validation. Thus, additional studies on different samples and with different measures are needed.

\section{Conclusion}

Although it purports to measure the construct of cognitive reflection or cognitive miserliness, it seems that the CRT does no such thing, at least not given the current conceptualization of cognitive reflection. The predictive power of the regular CRT seems to entirely stem from the fact that the CRT measures numerical and general mental abilities. This is corroborated by the lack of relationship between the CRT factor and the BBS factor, as well as the AOT factor, after partialling out the variance it shares with intelligence and numeracy, as well as by the very high correlations between the latent $\mathrm{g}$ factor and cognitive reflection. Conversely, it seems that the verbal cognitive reflection is a construct distinct from numeracy and intelligence. Unfortunately, similarly as the regular CRT, it failed to correlate with the belief bias and actively open-minded thinking factors. Therefore, judging from these results, neither of the measures seems to be able to assess the construct of cognitive reflection and their relationship with conceptually close measures stems from saturation of the CRT measures with general mental and numerical abilities. 


\section{Literature}

Bago, B., \& De Neys, W. (2017). Fast logic?: Examining the time course assumption of dual process theory. Cognition, 158, 90-109.

Baron, J., Scott, S., Fincher, K., \& Metz, S. E. (2015). Why does the Cognitive Reflection Test (sometimes) predict utilitarian moral judgment (and other things)?. Journal of Applied Research in Memory and Cognition, 4(3), 265-284.

Blacksmith, N., Yang, Y., Behrend, T. S., \& Ruark, G. A. (2019). Assessing the validity of inferences from scores on the cognitive reflection test. Journal of Behavioral Decision Making.

Bialek, M., \& Pennycook, G. (2018). The cognitive reflection test is robust to multiple exposures. Behavior research methods, 50(5), 1953-1959.

Brosnan, M., Hollinworth, M., Antoniadou, K., \& Lewton, M. (2014). Is empathizing intuitive and systemizing deliberative?. Personality and Individual Differences, 66, 39-43.

Bubić, A., \& Erceg, N. (2015). The relevance of cognitive styles for understanding individuals' cognitive functioning. Suvremena psihologija, 18(2), 159-173.

Campitelli, G., \& Gerrans, P. (2014). Does the cognitive reflection test measure cognitive reflection? A mathematical modeling approach. Memory \& cognition, 42(3), 434-447.

Campitelli, G., \& Labollita, M. (2010). Correlations of cognitive reflection with judgments and choices. Judgment and Decision Making, 5(3), 182-191.

Cokely, E.T., Galesic, M., Schulz, E., Ghazal, S., \& Garcia-Retamero, R. (2012). Measuring risk literacy: The Berlin Numeracy Test. Judgment and Decision Making, 7, 25-47.

Cokely, E. T., \& Kelley, C. M. (2009). Cognitive abilities and superior decision making under risk: A protocol analysis and process model evaluation. Judgment and Decision Making, $4(1), 20-33$.

Cronbach, L. J., \& Meehl, P. E. (1955). Construct validity in psychological tests. Psychological Bulletin, 52, 281-302.

De Neys, W. (2017). Bias, conflict, and fast logic: Towards a hybrid dual process future? In W. De Neys (Ed.), Dual Process Theory 2.0 (pp. 47-65). Oxon, UK: Routledge. 
Deppe, K. D., Gonzalez, F. J., Neiman, J. L., Jacobs, C., Pahlke, J., Smith, K. B., \& Hibbing, J. R. (2015). Reflective liberals and intuitive conservatives: A look at the Cognitive Reflection Test and ideology. Judgment \& Decision Making, 10(4).

Erceg, N., \& Bubić, A. (2017). One test, five scoring procedures: different ways of approaching the cognitive reflection test. Journal of Cognitive Psychology, 29(3), 381-392.

Evans, J. S. B., Barston, J. L., \& Pollard, P. (1983). On the conflict between logic and belief in syllogistic reasoning. Memory \& cognition, 11(3), 295-306.

Finucane, M. L., \& Gullion, C. M. (2010). Developing a tool for measuring the decisionmaking competence of older adults. Psychology and aging, 25(2), 271.

Frey, D., Johnson, E. D., \& De Neys, W. (2017). Individual differences in conflict detection during reasoning. The Quarterly Journal of Experimental Psychology, 71(5):1188-1208.

Gervais, W. M. (2015). Override the controversy: Analytic thinking predicts endorsement of evolution. Cognition, 142, 312-321.

Gino, F., \& Ariely, D. (2012). The dark side of creativity: original thinkers can be more dishonest. Journal of personality and social psychology, 102(3), 445.

Graffeo, M., Polonio, L., \& Bonini, N. (2015). Individual differences in competent consumer choice: the role of cognitive reflection and numeracy skills. Frontiers in psychology, 6 , 844.

Haigh, M. (2016). Has the standard cognitive reflection test become a victim of its own success? Advances in Cognitive Psychology,12,145-149.

Hoppe, E. I., \& Kusterer, D. J. (2011). Behavioral biases and cognitive reflection. Economics Letters, 110(2), 97-100.

Liberali, J. M., Reyna, V. F., Furlan, S., Stein, L. M., \& Pardo, S. T. (2012). Individual differences in numeracy and cognitive reflection, with implications for biases and fallacies in probability judgment. Journal of behavioral decision making, 25(4), 361-381. 
Lipkus, I. M., Samsa, G., \& Rimer, B. K. (2001). General performance on a Numeracy Scale among highly educated samples. Medical Decision Making, 21(1), 37-44. doi: 10.1177/0272989X0102100105

Markovits, H., \& Nantel, G. (1989). The belief-bias effect in the production and evaluation of logical conclusions. Memory \& Cognition, 17(1), 11-17.

Meyer, A., Zhou, E., \& Shane, F. (2018). The non-effects of repeated exposure to the Cognitive Reflection Test. Judgment and Decision making, 13(3), 246 - 259.

Newman, I. R., Gibb, M., \& Thompson, V. A. (2017). Rule-based reasoning is fast and belief-based reasoning can be slow: Challenging current explanations of belief-bias and base-rate neglect. Journal of experimental psychology: learning, memory, and cognition, 43(7), 1154.

Oechssler, J., Roider, A., \& Schmitz, P. W. (2009). Cognitive abilities and behavioral biases. Journal of Economic Behavior \& Organization, 72(1), 147-152.

Paxton, J. M., Ungar, L., \& Greene, J. D. (2012). Reflection and reasoning in moral judgment. Cognitive science, 36(1), 163-177.

Pennycook, G., Cheyne, J. A., Barr, N., Koehler, D. J., \& Fugelsang, J. A. (2015). On the reception and detection of pseudo-profound bullshit. Judgment and Decision making. 10(6), 549563

Pennycook, G., Cheyne, J. A., Koehler, D. J., \& Fugelsang, J. A. (2015a). Is the cognitive reflection test a measure of both reflection and intuition?. Behavior Research Methods, 48(1), 341348.

Pennycook, G., Cheyne, J. A., Seli, P., Koehler, D. J., \& Fugelsang, J. A. (2012). Analytic cognitive style predicts religious and paranormal belief. Cognition, 123(3), 335-346.

Pennycook, G., Fugelsang, J. A., \& Koehler, D. J. (2015b). Everyday consequences of analytic thinking. Current Directions in Psychological Science, 24(6), 425-432.

Pennycook, G., \& Rand, D. G. (2019). Cognitive reflection and the 2016 US Presidential election. Personality and Social Psychology Bulletin, 45(2), 224-239. 
Pennycook, G., \& Ross, R. M. (2016). Commentary: Cognitive reflection vs. calculation in decision making. Frontiers in Psychology, 7, 9.

Peters, E. (2012). Beyond comprehension: The role of numeracy in judgments and decisions. Current Directions in Psychological Science, 21(1), 31-35.

Podsakoff, P. M., MacKenzie, S. B., \& Podsakoff, N. P. (2016). Recommendations for creating better concept definitions in the organizational, behavioral, and social sciences. Organizational Research Methods, 19(2), 159-203.

Primi, C., Morsanyi, K., Chiesi, F., Donati, M. A., \& Hamilton, J. (2016). The development and testing of a new version of the cognitive reflection test applying item response theory (IRT). Journal of Behavioral Decision Making, 29(5), 453-469.

Reise, S. P. (2012). The rediscovery of bifactor measurement models. Multivariate behavioral research, 47(5), 667-696.

Reyna, V. F., Nelson, W. L., Han, P. K., \& Dieckmann, N. F. (2009). How numeracy influences risk comprehension and medical decision making. Psychological bulletin, 135(6), 943.

Royzman, E. B., Landy, J. F., \& Goodwin, G. P. (2014). Are good reasoners more incestfriendly? Trait cognitive reflection predicts selective moralization in a sample of American adults. Judgment and Decision Making, 9(3), 176-190.

Shaffer, J. A., DeGeest, D., \& Li, A. (2016). Tackling the problem of construct proliferation: A guide to assessing the discriminant validity of conceptually related constructs. Organizational Research Methods, 19(1), 80-110.

Shenhav, A., Rand, D. G., \& Greene, J. D. (2012). Divine intuition: Cognitive style influences belief in God. Journal of Experimental Psychology: General, 141(3), 423.

Shtulman, A., \& McCallum, K. (2014). Cognitive reflection predicts science understanding. In Proceedings of the Annual Meeting of the Cognitive Science Society (Vol. 36, No. 36).

Sinayev, A., \& Peters, E. (2015). Cognitive reflection vs. calculation in decision making. Frontiers in Psychology, 6, 532. 
Sirota, M., Kostovičová, L., Juanchich, M., Dewberry, C., \& Marshall, A. C. (2018, December 6). Measuring Cognitive Reflection without Maths: Developing and Validating the Verbal Cognitive Reflection Test. https://doi.org/10.31234/osf.io/pfe79

Stieger, S., \& Reips, U.-D. (2016). A limitation of the cognitive reflection test: Familiarity.PeerJ,4,e2395.

Stupple, E. J., Pitchford, M., Ball, L. J., Hunt, T. E., \& Steel, R. (2017). Slower is not always better: Response-time evidence clarifies the limited role of miserly information processing in the Cognitive Reflection Test. PloS one, 12(11), e0186404.

Thomson, K. S., \& Oppenheimer, D. M. (2016). Investigating an alternate form of the cognitive reflection test. Judgment and Decision making, 11(1), 99.

Thompson, V. A., Turner, J. A. P., \& Pennycook, G. (2011). Intuition, reason, and metacognition. Cognitive psychology, 63(3), 107-140.

Tishman, S., \& Andrade, A. (1996). Thinking dispositions: A review of current theories, practices, and issues. Cambridge, MA. Project Zero, Harvard University.

Toplak, M. E., West, R. F., \& Stanovich, K. E. (2011). The Cognitive Reflection Test as a predictor of performance on heuristics-and-biases tasks. Memory \& cognition, 39(7), 1275.

Toplak, M. E., West, R. F., \& Stanovich, K. E. (2014). Assessing miserly information processing: An expansion of the Cognitive Reflection Test. Thinking \& Reasoning, 20(2), 147-168.

Trippas, D., Kellen, D., Singmann, H., Pennycook, G., Koehler, D. J., Fugelsang, J. A., \& Dubé, C. (2018). Characterizing belief bias in syllogistic reasoning: A hierarchical Bayesian metaanalysis of ROC data. Psychonomic bulletin \& review, 25(6), 2141-2174.

Weller, J. A., Dieckmann, N. F., Tusler, M., Mertz, C. K., Burns, W. J., \& Peters, E. (2013). Development and testing of an abbreviated numeracy scale: A Rasch analysis approach. Journal of Behavioral Decision Making, 26(2), 198-212.

Welsh, M., Burns, N., \& Delfabbro, P. (2013). The cognitive reflection test: How much more than numerical ability?. In Proceedings of the Annual Meeting of the Cognitive Science Society (Vol. 35, No. 35). 


\section{Appendix}

\section{Numerical CRT}

1. A bat and a ball together cost 110 kunas. The bat costs 100 kunas more than the ball. How much does the ball cost?

2. If it takes 5 machines 5 minutes to make 5 widgets, how long would it take 100 machines to make 100 widgets?

3. In a lake, there is a patch of lily pads. Every day, the patch doubles in size. If it takes 48 days for the patch to cover the entire lake, how long would it take for the patch to cover half the lake?

4. Josip received a grade that is at the same time the fifteenth highest and the fifteenth lowest in the class. How many students are there in his class?

5. Simon decided to invest $\$ 8,000$ in the stock market one day early in 2008 . Six months after he invested, on July 17, the stocks he had purchased were down 50\%. Fortunately for Simon, from July 17 to October 17 , the stocks he had purchased went up $75 \%$. At this point, Simon has:
a. broken even in the stock market,
b. is ahead of where he began,
c. has lost money.

6. If 3 elves can wrap 3 toys in 1 hour, how many elves are needed to wrap 6 toys in 2 hours?

7. In an athletic team, tall athletes are three times more likely to win a medal than short athletes. This year the team has won 60 medals so far. How many of those medals were won by short athletes?

8. A square shaped garage roof with 6 meters long edge is covered with 100 tiles. How many tiles of the same size are covering a neighbouring roof, which is also square shaped, but with a 3 meters long edge?

9. There are two swimming pools in a swimming facility and in the summer they need to be filled with water. 100 liters of water are required to fill the cube-shaped pool. How many liters of water does it take to fill a cube-shaped pool but with a 3 times longer edges?

10. 25 soldiers are standing in a line 3 meters apart from each other. How many meters is the line long? 


\section{Verbal CRT}

1. It's a stormy night and a plane takes off from JFK airport in New York. The storm worsens, and the plane crashes-half lands in the United States, the other half lands in Canada. In which country do you bury the survivors?

2. A monkey, a squirrel, and a bird are racing to the top of a coconut tree. Who will get the banana first, the monkey, the squirrel, or the bird?

3. In a one-storey pink house, there was a pink person, a pink cat, a pink fish, a pink computer, a pink chair, a pink table, a pink telephone, a pink shower - everything was pink! What colour were the stairs probably?

4. How many of each animal did Moses put on the ark?

5. The wind blows west. An electric train runs east. In which cardinal direction does the smoke from the locomotive blow?

6. If you have only one match and you walk into a dark room where there is an oil lamp, a newspaper and wood - which thing would you light first?

7. Luke's father has three sons. The first two are called April and May. What is the name of the third son?

\section{Belief bias syllogisms}

1. Premise 1: All unemployed people are poor. Premise 2: Todorić* is not unemployed. Conclusion: Todorić* is not poor.

2. Premise 1: All flowers have petals. Premise 2: Roses have petals. Conclusion: Roses are flowers.

3. Premise 1: All Eastern countries are communist. Premise 2: Canada is not an Eastern country. Conclusion: Canada is not communist.

4. Premise 1: All things that have a motor need oil. Premise 2: Automobiles need oil. Conclusion: Automobiles have motors

* Todorić is a well-known Croatian rich businessman 